\title{
Estratificação do risco cardiovascular em pacientes hipertensos de um município do interior de Minas Gerais
}

\author{
Stratification of cardiovascular risk in hypertensive patients in a city in the interior of Minas \\ Gerais
}

\begin{abstract}
Estratificación del riesgo cardiovascular en pacientes hipertensos en una ciudad del interior de Minas Gerais
\end{abstract}

Frances Débora Ferreira de Deus ${ }^{1 *}$, Amanda Rocha Dorneles ${ }^{1}$, Natália de Fátima Gonçalves Amâncio .

\section{RESUMO}

Objetivo: avaliar o risco cardiovascular e seus agravos nos pacientes hipertensos do município de Patos de Minas- MG. Métodos: Trata-se de um estudo transversal, de natureza documental e abordagem quantitativa, que contou com a análise, após autorização do comitê de ética em pesquisa, de 46 prontuários, 23 homens e 23 mulheres, de 35 equipes de saúde da família, totalizando 1610 pacientes. Resultados: Após coleta dos dados, cada pessoa foi estratificada quanto ao risco cardiovascular em quatro fases. A primeira categorizou como alto risco 629 pacientes. A segunda, por meio do Escore de Risco Global, computou 146 pacientes em baixo, $390 \mathrm{em}$ intermediário e $273 \mathrm{em}$ alto risco. A terceira etapa enquadrou como alto risco 157 pacientes identificados anteriormente como risco intermediário. A quarta fase baseia-se na estratificação do Risco pelo Tempo de Vida e identificou 379 pacientes. Destes, $317 \mathrm{com}$ um fator de risco principal e $62 \mathrm{com}$ dois para apresentar eventos cardiovasculares de acordo com a exposição ao longo da vida. Conclusão: Foi significativo o número da população com risco cardiovascular alto e com agravantes passíveis de transformação, sugerindo uma oportunidade sem precedentes para alterar o curso das doenças crônicas não transmissíveis.

Palavras-chave: Doenças cardiovasculares, Doenças crônicas não transmissíveis, Fatores de risco cardiovascular.

\begin{abstract}
Objective: to evaluate cardiovascular risk and its injuries in hypertensive patients in the municipality of Patos de Minas- MG. Methods: It is a cross-sectional study, of documentary nature and quantitative approach, which counted on the analysis, after authorization by the research ethics committee, of 46 medical records, 23 men and 23 women, from 35 family health teams, totaling 1610 patients. Results: After data collection, each person was stratified according to cardiovascular risk in four phases. The first categorized 629 patients as high risk. The second, using the Global Risk Score, computed 146 patients below, 390 in intermediate and 273 at high risk. The third stage included 157 patients previously identified as intermediate risk as high risk. The fourth phase is based on life-time risk stratification and identified 379 patients. Of these, 317 with a major risk factor and 62 with two to present cardiovascular events according to lifelong exposure. Conclusion: There was a significant number of people at high cardiovascular risk and with aggravating factors that could be transformed, suggesting an unprecedented opportunity to change the course of chronic non-communicable diseases.
\end{abstract}

Keywords: Cardiovascular diseases, Chronic non-communicable diseases, Cardiovascular risk factors.

${ }^{1}$ Centro Universitário de Patos de Minas (UNIPAM), Patos de Minas - MG.

*E-mail: francesdeboradeus@hotmail.com

SUBMETIDO EM: 3/2021

PUBLICADO EM: 3/2021 


\section{RESUMEN}

Objetivo: evaluar el riesgo cardiovascular y sus lesiones en pacientes hipertensos en el municipio de Patos de Minas- MG. Métodos: Se trata de un estudio transversal, de carácter documental y abordaje cuantitativo, que contó con el análisis, previa autorización del comité de ética de la investigación, de 46 historias clínicas, 23 hombres y 23 mujeres, de 35 equipos de salud familiar, totalizando 1610 pacientes. Resultados: Después de la recopilación de datos, cada persona fue estratificada de acuerdo con el riesgo cardiovascular en cuatro fases. El primero clasificó a 629 pacientes como de alto riesgo. El segundo, utilizando la Puntuación Global de Riesgo, calculó 146 pacientes por debajo, 390 en intermedio y 273 en alto riesgo. La tercera etapa incluyó a 157 pacientes previamente identificados como de riesgo intermedio como de alto riesgo. La cuarta fase se basa en la estratificación del riesgo en tiempo de vida e identificó a 379 pacientes. De ellos, 317 con un factor de riesgo importante y 62 con dos para presentar eventos cardiovasculares según la exposición de por vida. Conclusión: Había un número importante de personas con alto riesgo cardiovascular y con factores agravantes que podrían transformarse, lo que sugiere una oportunidad sin precedentes para cambiar el curso de las enfermedades crónicas no transmisibles.

Palabras clave: Enfermedades cardiovasculares, Enfermedades crónicas no transmisibles, Factores de riesgo cardiovascular.

\section{INTRODUÇÃO}

O Sistema Único de Saúde (SUS), modificou o perfil sanitário do país representado, antes de sua implementação, por mazelas concernentes as doenças transmissíveis (DT), hoje pelas Doenças Crônicas não Transmissíveis (SOUZA FMF, et al., 2018). O supracitado é sentido por todas as sociedades e, por essa lógica, a Organização Mundial da Saúde (OMS) acordou vários compromissos a serem compartilhados constantes no plano de ação global que conta com nove metas voluntárias e 25 indicadores para prevenção e controle de diversas enfermidades. O acordado é que se alcance até 2025 uma redução de $25 \%$ dos óbitos incluindo os relacionados às Doenças Cardiovasculares (DCV) e seus agravos (WHO, 2014).

A agenda 2030 é um plano de ação global que visa a prosperidade do planeta por meio da erradicação da pobreza e foi adotada pelos 193 países membros da Organização das Nações Unidas ONU (2015). Ela propõe 17 objetivos de desenvolvimento sustentável (ODS), o terceiro relaciona-se à saúde e bem estar e engloba às DCV que juntos representam às áreas de intervenção capazes de afetar a saúde de todo o planeta e deverão ser alcançadas até 2030. Acompanhando as agendas acima referidas, o Instituto de Pesquisa Econômica Aplicada (IPEA), publicou em 2018 propostas de adequações destas metas à realidade brasileira visando à construção de indicadores capazes atingir este escopo.

Em referência às $\mathrm{DCV}$, redução significativa da taxa obituária pode ser evidenciada em diversos países que adotaram programas focados em sua prevenção principalmente pela identificação dos fatores de risco de maior prevalência na população (SIMÃO AF, et al., 2013). Segundo a pesquisa nacional de saúde de 2013, as DCNT se responsabilizam por $70 \%$ das mortes nacionais e apesar de evidenciado uma redução de 2,7\% no período de 2000-2013, Minas Gerais e o Rio de Janeiro possuem os maiores índices. As mortes prematuras, por ocasião de DCNT afetam principalmente indivíduos jovens, economicamente ativos e, apesar de não se restringirem a classes baixas, incide majoritariamente sobre esta população gerando mais pobreza e vulnerabilidade (MALTA DC, et al., 2018).

O Brasil possui grandes marcos no combate às DCNT e das ameaças modificáveis relacionadas a elas como o fumo, sedentarismo, obesidade e o abuso de álcool, alguns com resultados benéficos nos indicies nacionais (MALTA DC, et al., 2019). Apesar notável combate às mortes por DCV, segundo o editorial a cardiologia e o desenvolvimento sustentável ainda são necessárias ações que atuem sobre os agravos de maior impacto na população, com destaque para a Síndrome Metabólica (SM). De acordo com Ferreira ME (2016) o supracitado, é passível de prevenção e/ou tratamento e identificá-las precocemente tem o objetivo de conhecer as bases epidemiológicas, interpretar os riscos e intervir visando reduzir a prevalência e as complicações crônicas que tanto comprometem a qualidade e a sobrevida da população. 
Com este intento, a sociedade brasileira de cardiologia, já propunha mudanças na prática clínica arquitetando as bases necessárias ao enfrentamento das principais causas de adoecimento e de seus fatores de risco. Para atuar de maneira legitima sobre as DCNT, principalmente as que se relacionam às DCV, prevê a necessidade do diagnóstico precoce de pacientes com ameaça cardiovascular. Para alcançar tal objetivo propõe a prevenção e tratamento das comorbidades que com ela se associam e expõe a estratificação do risco em etapas composta por quatro fases (SIMÃO AF, et al., 2013).

A primeira é concernente à presença de doenças ateroscleróticas e de seus equivalentes que quando presentes categorizam automaticamente alto risco. A segunda, propõe a utilização do Escore de Risco Global, enquadrando os pacientes segundo o risco. A terceira identifica fatores agravantes e a presença de síndrome metabólica (SM) que categoriza como alto risco pacientes considerados como intermediários na etapa anterior. A quarta fase se baseia na estratificação do Risco pelo Tempo de Vida (RTV) capaz de aquilatar, a partir dos 45 anos, os indivíduos de baixo e intermediário risco acima classificados, motivando-os nas mudanças do estilo de vida (SIMÃO AF, et al., 2013).

Por serem, as Unidades Básicas de Saúde (UBS), a porta de entrada do SUS tornam-se o local ideal para estratificação do RCV visando resultados capazes de contribuir com o conhecimento epidemiológico, servir de cerne para estudos posteriores e legitimar a atuação efetiva dos profissionais. Justifica-se, para tanto, a identificação da população exposta e suas principais vulnerabilidades com intento de adaptar tratamentos e equilibrar fatores que possam cooperar com o reestabelecimento salutar da região. Para tanto objetiva-se auxiliar o enfrentamento do grande desafio contemporâneo concernente à redução da mortalidade por causas evitáveis, por meio do reconhecimento dos indivíduos com RCV e seus agravos, bem como as áreas com maior representatividade dos mesmos.

\section{MÉTODOS}

Trata-se de um estudo transversal, de natureza documental e abordagem quantitativa, com a utilização de dados secundários constantes em prontuário e procedimentos técnicos de pesquisa bibliográfica, buscando utiliza-los para classificação do risco cardiovascular.

A população de estudo foi constituída pelo prontuário dos moradores pertencentes à área de abrangência geográfica de um município do interior de Minas Gerais, nas 20 Unidades Básicas de Saúde compostas por 35 Equipes de Saúde da Família (ESF). A amostra incluiu a análise documental de 46 pacientes, sendo 23 homens e 23 mulheres, para cada equipe, na faixa etária de 45 anos ou mais, previamente diagnosticados com hipertensão arterial sistêmica, perfazendo um total de 1.610 prontuários. Foram excluídos da amostra áreas correspondentes ao território rural, os pacientes que não possuíam em seus registros as informações necessárias para classificação do escore de risco.

Os instrumentos de coleta de dados para avaliação do RCV são: prontuários médicos dos pacientes hipertensos e seus exames laboratoriais. Escore de Risco Global, o Escore de Risco pelo Tempo de Vida e os critérios diagnósticos de SM (SIMÃO AF, et al., 2013; ABESO, 2016).

Após coleta dos dados em prontuário, cada paciente cadastrado foi estratificado quanto ao RCV em quatro fases. Na primeira foram observados a presença de doença aterosclerótica significativa ou de seus equivalentes constantes nos critérios de identificação de pacientes com alto risco de eventos coronarianos da I Diretriz Brasileira de Prevenção Cardiovascular, desde que documentadas em prontuário e, quando presentes, considerados automaticamente de alto risco.

A segunda fase representa o Escore de Risco Global (ERG) encontrado na diretriz supracitada, sendo de baixo risco aqueles com $<5 \%$ de probabilidade de apresentarem eventos cardiovasculares nos últimos 10 anos e reclassificados com risco intermediário quando presente história de DCV prematura. Considerados de risco intermediário os homens que apresentaram probabilidade de qualquer evento supracitado $\geq 5 \%$ e $\leq 20 \%$ e mulheres $\geq$ a $5 \%$ e $\leq$ a $10 \%$ e, de alto risco, os homens que obtiverem risco calculado $>20 \%$ e mulheres $>10 \%$.

$\mathrm{Na}$ terceira fase identificamos critérios de Síndrome Metabólica e história familiar prematura de doença arterial coronariana como fatores para determinar alto risco nos pacientes considerados como intermediários na etapa anterior. 
A quarta fase representa o algoritmo de estratificação de risco pelo tempo de vida (RTV) nos maiores de 45 anos, de ambos sexos, classificados acima como de baixo e de intermediário risco. Foram evidenciados os fatores de risco ótimos, não ótimos, elevados e principais, estimando o percentual de pacientes com risco de eventos cardiovasculares fatais e não fatais de acordo com a exposição ao longo da vida.

Além do exposto, foram calculados os IMC dos pacientes de 45 a 59 anos de acordo com a I Diretriz Brasileira de Diagnóstico e Tratamento da Síndrome Metabólica e a partir de 60 anos, como prevê a Caderneta de Saúde da Pessoa Idosa, sendo a obesidade e o sobrepeso considerados como fatores de risco adicionais (SOCIEDADE BRASILEIRA DE HIPERTENSAO, et al., 2005; MINISTÉRIO DA SAÚDE, 2014).

Para elaborar a construção teórica foram utilizados trabalhos disponíveis em base de dados como: Medical Literature Analysis and Retrieval System Online (MEDLINE), Scientific Eletronic Library Online (SCIELO), Literatura Latino-Americana e do Caribe e Ciências da Saúde (LILACS) e Google Scholar. Os descritores utilizados foram: DCV, DCNT e fatores de RCV. Foram consultadas a biblioteca virtual do Ministério da Saúde (BVSMS), Instituto de Pesquisa Econômica e Aplicada (IPEA) e Instituto Brasileiro de Geografia e Estatística (IBGE). Os dados foram utilizados para análise estatística de média, desvio padrão, porcentagem e frequência utilizando o programa SPSS e para confecção de tabelas e gráficos, utilizou-se o Microsoft Excel. O projeto deste estudo foi aprovado pelo Comitê de Ética em Pesquisa do Centro Universitário de Patos de MinasUNIPAM (Parecer ํㅜ 4.049.605 e CAAE: 20284519.0.0000.5549).

\section{RESULTADOS E DISCUSSÃO}

Os resultados relatados abaixo referem-se à coleta final dos dados, correspondentes a 35 Equipes de Saúde da Família de um município do interior de Minas Gerais, previamente identificadas, perfazendo um total de 1610 prontuários. Destes 1434 foram selecionados e 176 excluídos, por não atenderem o escopo deste trabalho. A amostra está composta por 724 prontuários femininos e 710 prontuários masculinos, todos submetidos à avaliação do RCV. A identificação do perfil dos pacientes com este risco torna-se relevante em virtude da caracterização do público estudado, de modo a nortear possíveis condutas clínicas e terapêuticas por parte dos profissionais atuantes nas UBS, bem como produzir evidências científicas.

A partir dos dados investigados foram categorizados na primeira fase com alto risco cardiovascular 322 pacientes do sexo feminino, e 307 do sexo masculino apresentando diferentes perfis para as principais manifestações ateroscleróticas (Tabela 1).

Tabela 1 - Perfil dos pacientes com alto risco cardiovascular (RCV).

\begin{tabular}{lcccc}
\hline & \multicolumn{2}{c}{ Feminino } & \multicolumn{2}{c}{ Masculino } \\
\cline { 2 - 5 } & $\mathbf{n}$ & $\mathbf{0}$ & $\mathbf{n}$ & $\%$ \\
\hline Diabetes Mellitus (DM) & 172 & $53,40 \%$ & 134 & $43,70 \%$ \\
Doença Renal Crônica (DRC) & 28 & $8,70 \%$ & 25 & $8,10 \%$ \\
DM+DRC & 35 & $10,90 \%$ & 17 & $5,60 \%$ \\
Diversas Manifestações Ateroscleróticas & 87 & $27,00 \%$ & 131 & $42,60 \%$ \\
\hline TOTAL & $\mathbf{3 2 2}$ & $\mathbf{4 4 , 4 7 \%}$ & $\mathbf{3 0 7}$ & $\mathbf{4 3 , 2 4 \%}$ \\
\hline
\end{tabular}

Fonte: Deus FDF, et al., 2021.

De acordo com Mafra F e Oliveira H (2008) complicações cardiovasculares depende de uma somatória de fatores e, apesar de evolução lenta, o objetivo de calcular o risco é a prevenção. Quando já estabelecida, a intenção é minimizar os riscos principalmente pela manutenção de níveis pressóricos e glicêmicos dentro da normalidade. A hipertensão, segundo Malachias MVB, et al. (2016), acomete cerca de 32,5\% dos indivíduos adultos do país, representa $50 \%$ das mortes por DCV e está implicada no aumento do número de hospitalizações, na queda da produtividade e da renda familiar.

Ainda segundo Malachias MVB, et al. (2016), por se tratar de uma afecção silenciosa, permite o desenvolvimento de complicações principalmente quando em concomitância com DM e doença 
aterosclerótica. Dos 1434 prontuários analisados 629 , apontados na tabela acima, apresentam hipertensão associada a outros fatores de RCV determinando uma prevalência de $43,8 \%$ de pacientes considerados alvos de intervenções que de alguma forma visem minimizar a progressão da moléstia e seus agravos. Infelizmente, segundo a Diretriz da Sociedade Brasileira de Diabetes, muitos governos e profissionais ainda não se conscientizaram de sua relevância o que prejudica seu manejo e a implementação de medidas para o controle metabólico destes pacientes (SOCIEDADE BRASILEIRA DE DIABETES, 2020).

O relatório de status global sobre doenças não transmissíveis da OMS (2014), acordou nove metas globais voluntárias sendo a maior delas a proposta de redução de $25 \%$ dos óbitos prematuros por estes agravos até 2025. O refreio relativo da incidência de diabetes e hipertensão contemplam outras duas metas e alcança-las significa atenuar os portadores de tais riscos. De acordo com a pesquisa nacional de saúde IBGE (2014), o exposto representa 70\% das causas de mortes evitáveis no país, uma ameaça à economia e à qualidade de vida.

A população analisada reflete os usuários da atenção primária, local ideal para alterar desfechos e reduzir os finamentos. Em um contexto de multidisciplinaridade individual e familiar, o objetivo é orientar, prevenir e recuperar a saúde destes indivíduos. A amostra desta primeira fase apresentou um número significativo com alto risco e a partir deste perfil, o intendo é contribuir com a qualidade do acompanhamento e acesso aos medicamentos necessários para tratá-las.

Declarado por Mesquita CT, et al. (2018), diversos países alcançam expressivos resultados por meio da identificação do RCV e da criação de programas focados em sua prevenção. No Brasil ainda são necessárias ações efetivas de promoção à saúde que atuem de forma mais consistente na redução dos agravos de maior impacto. O enfoque deste estudo é proporcionar, pela estratificação do RCV, ferramentas para ações dentro da atenção primária com foco nas necessidades e perfil populacional. O exposto na tabela infere a somatória de DCNT encontradas e que, por seu caráter devastador, por si já justificaria uma investigação precoce e apesar de não suplantar a cura possibilita intervenções capazes de alterar seu curso e contribui para repercussões futuras na saúde.

Os pacientes que não se enquadraram nas condições de alto risco na primeira fase, foram adequados no Escore de Risco Global, segundo recomendado pela I Diretriz Brasileira de Prevenção Cardiovascular, correspondendo à segunda fase (SIMÃO AF, et al., 2013). Por este escore, amoldaram-se na categoria de baixo, intermediário e alto risco, 100, 182 e 122 mulheres e 46, 208 e 151 homens, respectivamente. Todos com risco calculado de ocorrência de um primeiro ou um novo evento cardiovascular em 10 anos (Gráfico 1).

Gráfico 1 - Estratificação da amostra do estudo, conforme Escore de Risco Global.

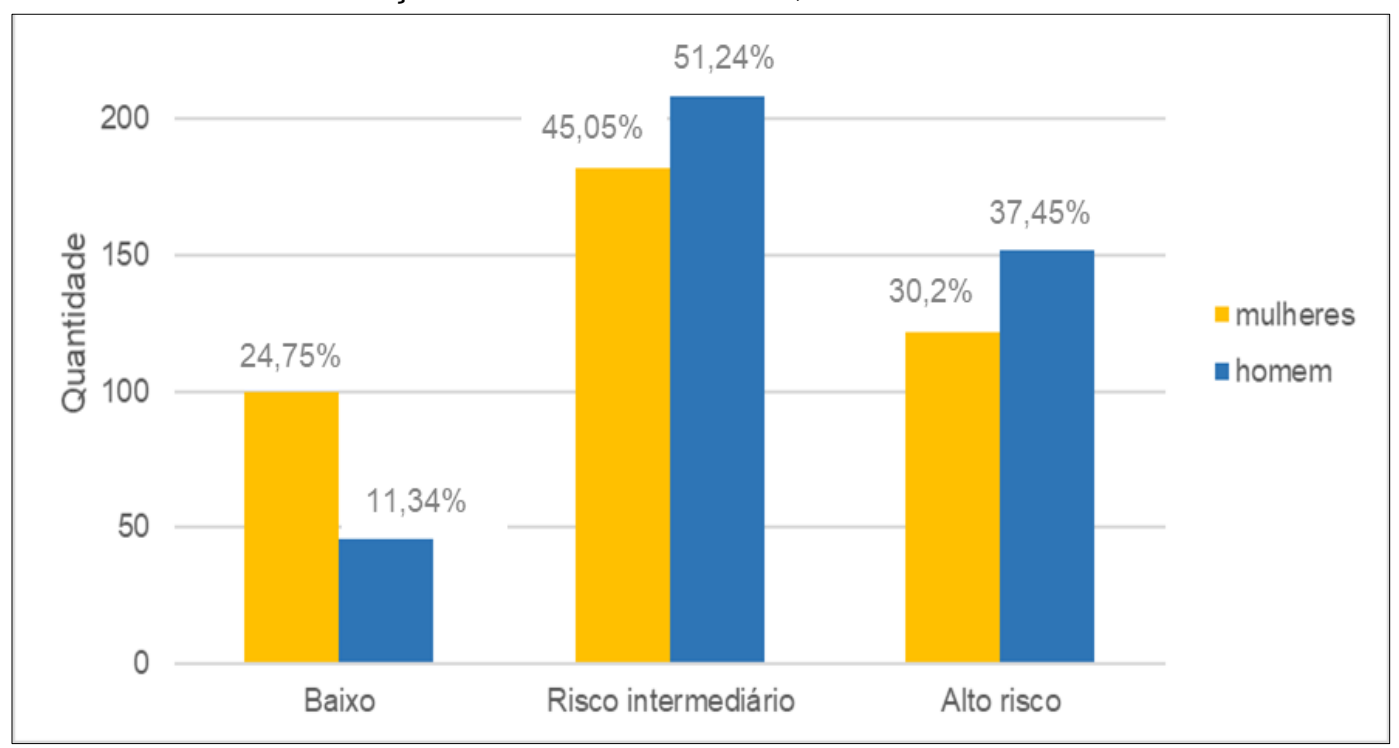

Fonte: Deus FDF, et al., 2021. 
Por meio de Simão AF, et al. (2013); e, arquitetando as bases necessárias ao enfrentamento das principais causas de morte por DCV e de seus agravos prevê a necessidade do diagnóstico destes pacientes para prevenção e tratamento precoce. Afirma que existem diversos algoritmos consagrados na literatura, dentre eles o ERG e o RTV, ambos utilizados no presente estudo para melhor aproximação de risco. As principais condições analisadas pelo escore de risco cardiovascular são imutáveis como sexo, idade e hereditariedade, entretanto parte significativa das ameaças que se relacionam com as mortes por este dano são passíveis de controle.

Os grupos de baixo e intermediário risco representam $66,25 \%$ da amostra submetida à segunda fase e, quando comparados com os de alto risco, possuem melhores condições clínicas pela menor associação de comorbidades. Expressam maior facilidade de alcançar metas propostas por intervenção no estilo de vida como atividades e alimentação e consequentemente menor necessidade de tratamento medicamentoso. $\mathrm{O}$ anunciado reforça a importância do uso de ferramentas capazes de fornecer um diagnóstico precoce para 0 seu controle e segmento.

Nas últimas décadas, o Brasil contou com transformações da saúde pública, com a implementação de ações em vigilância, amplificação do acesso aos cuidados e cobertura de tratamentos. O impresso, ONU (2015), abarca o documento que contempla as principais áreas capazes de assegurar proteção duradoura ao planeta aplicáveis nas diferentes realidades e contem 17 objetivos (ODS), nos quais se encontram indicadores e metas para mudanças na qualidade de vida e na saúde mundial. O terceiro alvo, se concatena à saúde e bem-estar com o objetivo de reduzir as mortes prematuras por doenças não transmissíveis admitindo entre elas as DCV, seus agravos e reafirmando a necessidade de identificação precoce.

A estratificação do RCV auxilia no tratamento, no prognóstico e no conhecimento dos indivíduos com propensão a complicações (MALACHIAS MVB, et al., 2016). Inferimos com o gráfico 1 que 146 pacientes tiveram, com esta ferramenta, um rastreamento oportuno significando a possibilidade de mudança principalmente por meio de uma alimentação saudável e atividade física. A amostra classificada como risco intermediário, representada por 390 pacientes, pode vir a ser considerada de baixo risco caso adotem as mesmas intervenções e as aliem ao tratamento medicamentoso evitando lesões subclínicas e de órgãos alvo. Os 273 restantes são considerados de alto risco e a intervenção neste grupo, além da conscientização da doença é evitar a progressão das lesões e reduzir o risco dos eventos CV.

Segundo a WHO (2014), a disponibilidade de orçamento, tecnologias e medicamentos para prevenção de diversas DCNT pode ser adaptada às diversas realidades e contextos mundiais não havendo razão para qualquer país atrasar tais mudanças. Baseado nesta ideia, o Instituto de Pesquisa Econômica Aplicada (IPEA), publicou em 2018 propostas de adequações destas metas à realidade brasileira visando à construção de indicadores nacionais aptos a atingir tal escopo. Por meio do ERG conseguimos calcular o risco de ocorrência de um primeiro ou um novo evento $\mathrm{CV}$ em 10 anos para todos a um baixo custo, o que facilita sua aplicação, reconhecimento, intervenção e controle precoce contribuindo com a meta da OMS em reduzir em $25 \%$ a mortalidade geral por esses agravos.

Dos pacientes de risco intermediário pelo Escore de Risco Global, 71 mulheres e 86 homens foram reclassificados na terceira fase como de alto risco por apresentarem síndrome metabólica, considerado fator agravante pela I Diretriz Brasileira de Prevenção Cardiovascular (SIMÃO AF, et al., 2013) (Tabela 2).

Tabela 2 - Perfil dos pacientes com síndrome metabólica e alto RCV.

\begin{tabular}{lcccc}
\hline & \multicolumn{2}{c}{ Mulheres } & \multicolumn{2}{c}{ Homens } \\
\cline { 2 - 5 } & $\mathbf{n}$ & $\%$ & $\mathbf{n}$ & $\%$ \\
\hline Com Síndrome Metabólica & 71 & $39,01 \%$ & 86 & $41,35 \%$ \\
Sem Síndrome Metabólica & 111 & $60,99 \%$ & 122 & $58,65 \%$ \\
\hline Total & $\mathbf{1 8 2}$ & $\mathbf{1 0 0 , 0 0 \%}$ & $\mathbf{2 0 8}$ & $\mathbf{1 0 0 , 0 0 \%}$ \\
\hline
\end{tabular}

Fonte: Deus FDF, et al., 2021. 
De acordo com a I Diretriz de Prevenção Cardiovascular 2013, o Brasil assumiu o compromisso de prevenção e de cuidados necessários ao enfrentamento das DCNT bem como de suas ameaças, principalmente as modificáveis. A sociedade brasileira de cardiologia por meio da Faludi AA, et al. (2017) propôs mudanças na prática clínica capazes de influenciar nos desfechos salutares concernentes às DCV. Merece destaque pela grande prevalência e alto risco cardiovascular a SM que engloba a hipertensão arterial, dislipidemias, diabetes mellitos e circunferência abdominal, refletida em $40,25 \%$ da amostra total investigada na terceira etapa.

A SM agrava ao mesmo tempo que é passível de prevenção e/ou tratamento e permite, ao conhecer a população que a externa, a possibilidade de intervenção e mudança nos indicadores de saúde. A prevenção está na própria definição da síndrome que, por meio de atividade física regular, mudança alimentar e adesão ao tratamento medicamentoso é capaz de intervir na história natural de diversas doenças. O exposto pode ser alcançado pela redução da circunferência abdominal, controle da glicemia, do perfil lipídico e da pressão arterial, beneficiando neste caso 390 pacientes com reflexo, a longo prazo, na saúde da população circunscrita (SIMÃO AF, et al., 2013).

Na quarta fase, os que continuaram com risco intermediário na fase anterior ou foram categorizados como de baixo risco na segunda submeteram-se a estratificação do RTV. Encontramos 176 mulheres e 141 homens com 01 fator de risco principal e 35 mulheres e 27 homens com dois ou mais destes fatores. Seus riscos médios de apresentarem eventos relacionados às DCV e aterosclerótica (Tabela 3).

Tabela 3 - Risco de eventos cardiovasculares pelo tempo de vida para homens e mulheres.

\begin{tabular}{|c|c|c|c|}
\hline & & \multicolumn{2}{|c|}{ Risco percentual (intervalo de confiança 95\%) } \\
\hline & & $\begin{array}{c}1 \text { Fator de risco } \\
\text { principal }\end{array}$ & $\begin{array}{l}2 \text { ou mais Fatores de } \\
\text { risco principais }\end{array}$ \\
\hline \multirow{5}{*}{ Homens } & DAC fatal ou IAM não fatal & $34,0(30,4-37,6)$ & $42,0(37,6-46,5)$ \\
\hline & AVC fatal ou não fatal & $8,4(7,5-9,4)$ & $10,3(9,0-11,7)$ \\
\hline & Morte por DCV & $20,7(19,4-22,2)$ & $32,5(30,5-34,5)$ \\
\hline & $\begin{array}{l}\text { Total de eventos relacionados a DCV } \\
\text { aterosclerótica }\end{array}$ & $39,6(35,7-43,6)$ & $49,5(45,0-53,9)$ \\
\hline & Total de indivíduos & 141 & 27 \\
\hline \multirow{5}{*}{ Mulheres } & DAC fatal ou IAM não fatal & $12,7(10,3-15,0)$ & $21,5(17,5-25,5)$ \\
\hline & AVC fatal ou não fatal & $9,1(7,9-15,9)$ & $11,5(9,5-13,5)$ \\
\hline & Morte por DCV & $11,2(9,9-12,5)$ & $21,9(19,4-24,5)$ \\
\hline & $\begin{array}{l}\text { Total de eventos relacionados a DCV } \\
\text { aterosclerótica }\end{array}$ & $20,2(17,2-23,2)$ & $30,7(26,3-35,0)$ \\
\hline & Total de indivíduos & 176 & 35 \\
\hline
\end{tabular}

Em conformidade com BRASIL (2003), diversas ações em atenção básica dirigidas a grupos específicos da população, incluindo as DCV, já foram estruturadas prevendo impacto positivo nos indicadores de hospitalizações e morbimortalidade. O interessante da estratificação de RTV se encontra na possibilidade de identificar os pacientes com risco ao longo do tempo e prevenir sua ocorrência. Para trabalhar em consonância com a lei e com desafio contemporâneo no intento de reduzir as mortes por DCNT é essencial aos profissionais aptos a atuar na porta de entrada o encargo de dominar não apenas as mazelas mais também de envolver a população a elas exposta e traçar metas.

Para WHO (2014), o mundo chegou a um ponto decisivo na história das DCNT com oportunidades sem precedentes para alterar seu curso com a promoção de saúde. Segundo Malachias MVB, et al. (2016), os pacientes devem inteirar-se de suas doenças, assumir a responsabilidade por sua saúde e se comprometer 
a melhorá-la. Devem ser instruídos sobre os benefícios do autocuidado, da alimentação saudável, da importância da atividade física, do controle de peso e da cessação do tabagismo, pontos sabidamente importantes para redução do RCV e lesões de órgãos alvo.

Pela análise dos agravos, sobrepeso, obesidade e uso de tabaco, encontramos, nas mulheres, um total de 395, 113 e 121 e nos homens de 378, 100 e 168, respectivamente (Gráfico 2).

Gráfico 2 - Prevalência dos agravantes relacionados à DCV.

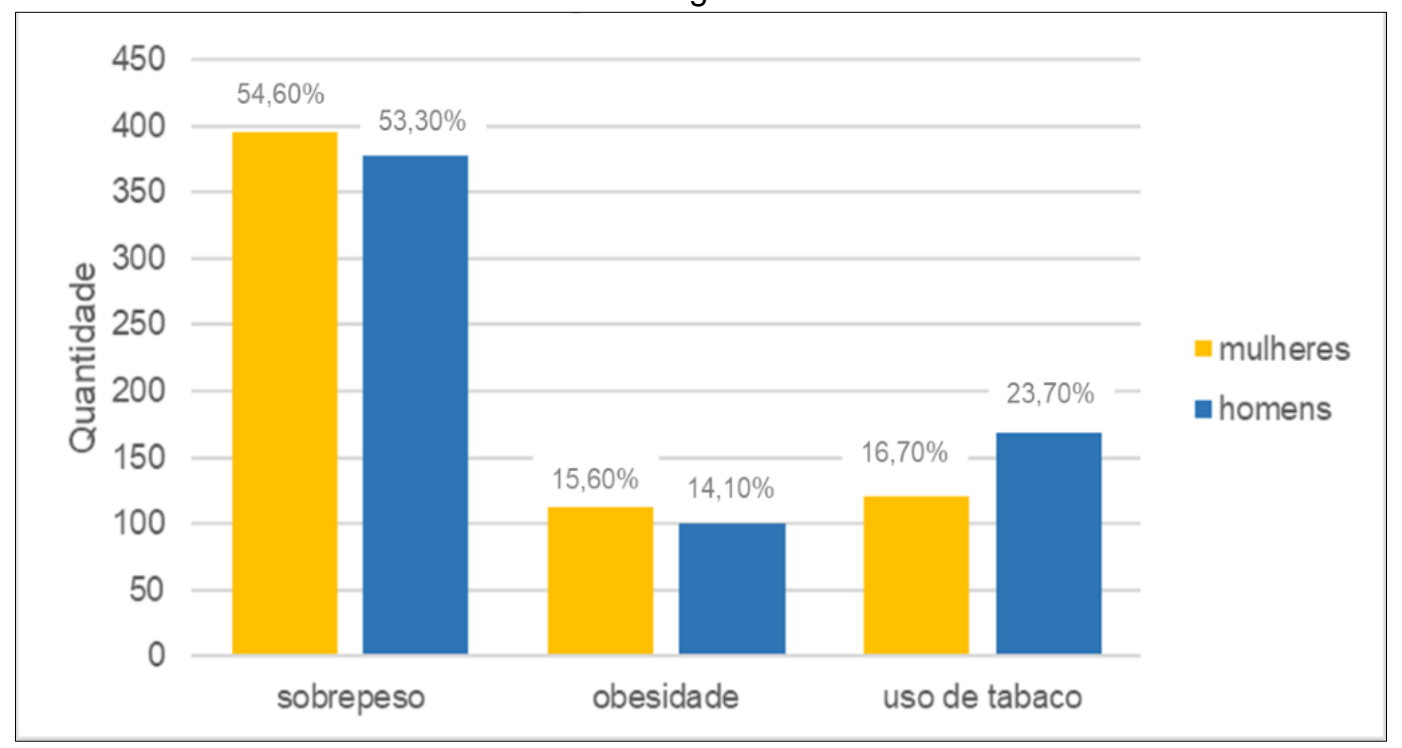

Fonte: Deus FDF, et al., 2021.

A redução do uso abusivo do álcool, do tabaco, do sal/sódio, do sedentarismo, também faz parte das metas do relatório de status global já citado, no intuito de reduzir as mortes por causas evitáveis. De acordo com Simão AF, et al. (2013) os pontos acima investigados, quando presentes, além de aumentar o risco cardiovascular são contribuintes diretos da SM, que segundo dados da BRASIL (2019), vem aumentando na população brasileira. A identificação prévia destes pacientes possibilita intervenções capazes de modificar a história natural da doença e a simples implementação de medidas nutricionais, o incentivo à prática regular de atividade física e as mudanças dos maus hábitos, como parte do plano terapêutico, é capaz de atenuar a probabilidade de desfechos futuros ruins.

As DCNT são agravos multifatoriais, de desenvolvimento lento e pauta de grande preocupação contemporânea, sendo que elencar o risco e inventariar a prevalência das principais doenças a elas conectadas, faculta a transformação na qualidade de vida da população. O gráfico acima revela 1275 pacientes que externaram agravantes de RCV que, segundo Sintjago EM (2019), aumentam a morbimortalidade, o que é corroborado por Pinho PM (2014) que sugere agravo no prognóstico e impacto social e econômico ao país. Tal informe reforça a importância da utilização de ferramentas como o cálculo do RCV nos diversos cenários de atuação das ESF, para a ingerência precoce, controle de comorbidades e o segmento contínuo destes pacientes.

\section{CONCLUSÃO}

Este estudo demostrou ser significativo o número da população com risco cardiovascular alto e com agravantes passíveis de transformação como o fumo e a obesidade. Isso sugere uma oportunidade sem precedentes para alterar o curso das doenças crônicas não transmissíveis, visto que a intervenção precoce sabidamente interfere na história natural da doença e a demora na tomada de decisão resulta em maior custo com a saúde. Reconhecer as áreas de maior vulnerabilidade, auxilia na atuação das ESF e na elaboração de intervenções eficientes capazes de reduzir os riscos e cooperar com o reestabelecimento salutar da comunidade. Mais pesquisas nesse campo são necessárias para o conhecimento epidemiológico das doenças cardiovasculares e dos seus determinantes sociais e ambientais na população no município. 


\section{REFERÊNCIAS}

1. ABESO. DIRETRIZES BRASILEIRAS DE OBESIDADE/ABESO- Associação Brasileira para o Estudo da Obesidade e da Síndrome Metabólica, São Paulo, 2016; 4:188p.

2. BRASIL. Conselho Nacional de Secretários de Saúde. Legislação do SUS. Brasília: CONASS, 2003. 604p.

3. BRASIL, Ministério da Saúde. Secretaria de Vigilância em Saúde, Departamento de Vigilância de Doenças e Agravos não transmissíveis e Promoção da Saúde. Brasília; 2020, 8p.

4. BRASIL. Ministério da Saúde. Secretaria de vigilância em Saúde. Departamento de Vigilância de Doenças e Agravos não transmissíveis e Promoção da saúde. Vigitel Brasil 2019: vigilância de fatores de risco e proteção para doenças crônicas por inquérito telefônico. Brasília: Ministério de Saúde, 2019. 139 p.

5. FALUDI AA, et al. Sociedade Brasileira de Cardiologia. Atualização da Diretriz Brasileira de Dislipidemia e Prevenção da Aterosclerose. Arquivos Brasileiros de cardiologia, 2017; 109(2): 1-91.

6. FERREIRA ME. Síndrome metabólica e doenças cardiovasculares: do conceito ao tratamento. Arq. Catarin Med. 2016; 45:95-109.

7. INSTITUTO DE PESQUISA ECONÔMICA E APLICADA, ODS: Metas Nacionais dos Objetivos de Desenvolvimento Sustentável, agenda 2030 p. 546, 2018.

8. MAFRA F, OLIVEIRA H. Avaliação do risco cardiovascular - metodologia e suas implicações na prática clínica. Rev Port Clin Geral, 2008; 24: 391-400.

9. MALACHIAS MVB, et al. 7ª Diretriz Brasileira de Hipertensão Arterial. Arquivos Brasileiros de Cardiologia, Rio de Janeiro. 2016. 107(3):1-104.

10. MALTA DC, et al. Mortes Evitáveis no Sistema Único de Saúde na População Brasileira, entre 5 e 69 anos, 2000 2013. Revista Brasileira de Epidemiologia. 2018, 21: 1-15.

11. MALTA DC, et al. Probabilidade de Morte Prematura por Doenças Crônicas não Transmissíveis, Brasil e Regiões, Projeções para 2025. Revista Brasileira de epidemiologia, 2019; 22: 1-13.

12. MESQUITA CT, LEÃO M. A. Cardiologia e o Desenvolvimento Sustentável. International Journal of Cardiovascular Sciences. 2018; 31(1):1-3.

13. MINISTÉRIO DA SAÚDE. Caderneta de Saúde da Pessoa Idosa. 3aed. Brasília-DF. 2014. 58p.

14. ONU. United Nations Organization. Transforming our World: The 2030 Agenda for Sustainable Development. 2015.

15. PESQUISA NACIONAL DE SAÚDE 2013/IBGE, Percepção do Estado de Saúde, Estilos de Vida e Doenças Crônicas, 2014; p. 181.

16. PINHO PM, et al. Síndrome metabólica e sua relação com escores de risco cardiovascular em adultos com doenças crônicas não transmissíveis Rev. Soc. Bras. Clín. Méd; 2014. 12(1) -9p.

17. SIMÃO AF, et al. Sociedade Brasileira de Cardiologia. I Diretriz Brasileira de Prevenção Cardiovascular. Arq Bras Cardiol. 2013; 101(6):1-63.

18. SINTJAGO EM, et al. Coocorrência de Fatores de Risco para Doenças Cardiometabólicas: Alimentação Não Saudável, Tabaco, Álcool, Estilo de Vida Sedentário e Aspectos Socioeconômicos. Arq. Bras. Cardiol. 2019; 113 (4): $710-711$.

19. SOCIEDADE BRASILEIRA DE DIABETES. Diretrizes da Sociedade Brasileira de Diabetes 2019-2020: 13p.

20. SOCIEDADE BRASILEIRA DE HIPERTENSAO, et al. I Diretriz Brasileira de Diagnóstico e Tratamento da Síndrome Metabólica. Arq. Bras. Cardiol., São Paulo, v. 84, supl. 1, p. 3-28, 2005.

21. SOUZA MFM, et al. Transição da Saúde e da Doença no Brasil e nas Unidades Federadas durante os 30 anos do Sistema Único de Saúde. Ciência e Saúde Coletiva, 2018; 23(6):1737-1750.

22. WHO. WORLD HEALTH ORGANIZATION. Global status report on noncommunicable diseases. Genebra: World Health Organization; 2014, 302p. 